\title{
La experiencia de la Chakra, como ambiente de aprendizaje, en el Centro de Educación Infantil de Innovación UNAE
}

\section{Fernanda Acosta ${ }^{a}$, Valeria Fárez Román ${ }^{b}$ y Byron Cevallos Trujillo ${ }^{c}$}

afernanda.acosta@unae.edu.ec,_bclaudia.farez@unae.edu.ecｙｃbyron.cevallos@unae.edu.ec Universidad Nacional de Educación

\begin{abstract}
Chakra is the ancestral agricultural space, with a type of associative crop from the Andean region. It is native from the kichwa/quechua peoples and it promotes the construction of knowledge from the collective wisdom (Arévalo, 2017). This transdisciplinary innovation experience proposes chakra as an intercultural collaborative learning environment and a pedagogical junction point, in the interactions between Universidad Nacional de Educación (UNAE), the UNAE Preschool Centre for innovation, the family and the community. In this communication we present the context in which we have constructed this proposal, the educative processes we have carried out within the learning community inside the Preschool Centre and the pedagogical actions we have developed from the University. The experience has generated a meaningful experiential learning for the different involved actors who participate on the Chakra maintenance, through the exchange of knowledge and the development of links within them and with the environment.
\end{abstract}

\section{Keywords:}

chakra; educational innovation; exchange of knowledge; iintercultural learning environment; early childhood education.

\section{Resumen}

La chakra es el espacio agrícola ancestral, con un tipo de cultivo asociado, propio de la región andina, proveniente de los pueblos kichwas/quechuas, que propicia la construcción del conocimiento desde los saberes colectivos (Arévalo, 2017). Esta experiencia transdisciplinaria de innovación plantea a la chakra escolar, como un ambiente de aprendizaje colaborativo intercultural y como núcleo pedagógico articulador, en las interacciones de la Universidad Nacional de Educación (UNAE), el Centro de Educación Infantil (CEI) de innovación anexo a la UNAE, la familia y la comunidad circundante. En esta comunicación presentamos los distintos contextos en los que hemos tejido esta propuesta, los procesos educativos ejecutados por la comunidad de aprendizaje dentro del CEI y las acciones pedagógicas que hemos realizado desde la docencia en la UNAE. La experiencia, ha generado un aprendizaje vivencial significativo para las y los diferentes actores que participan de su cuidado, a través del intercambio de saberes y la creación de vínculos entre ellas, ellos y a su vez con el entorno natural.

Palabras clave: chakra; innovación educativa; intercambio de saberes; ambiente de aprendizaje intercultural; educación inicial. 


\section{Introducción}

Se conoce como chakra al tipo de siembra asociado, proveniente de los pueblos kichwas/quechuas de los Andes (Ecuador, Perú, Bolivia y Colombia). Se realiza entre plantas que no se aíslan para su crecimiento, sino que, al contrario, se complementan unas a otras. Según Arévalo (2017), dentro de la chakra puede haber sembríos de varios tipos de plantas, siendo imprescindibles dentro de ella, el maíz y el frijol. El concepto de chakra, como práctica agrícola ancestral, pervive actualmente en los campos andinos. Se propone como un escenario educativo ideal, para la trasndisciplinariedad, el amor a la Naturaleza y el intercambio de saberes, en un contexto de igualdad entre los conocimientos occidentales y ancestrales.

Además, la chakra escolar, a diferencia de una huerta, establece un enfoque de interculturalidad, en la acción socioeducativa. Para Catherine Walsh, quien ha dedicado su investigación con pueblos indígenas de Ecuador, comprende a la interculturalidad, desde una perspectiva crítica, como "herramienta y proceso" que se recrea en la sociedad, desde la demanda de la subalternidad, en contraposición a la hegemonía cultural.

La interculturalidad entendida críticamente aún no existe, es algo por construir. Por eso, se entiende como una estrategia, acción y proceso permanente de relación y negociación entre, en condiciones de respeto, legitimidad, simetría, equidad e igualdad. Pero aún más importante es su entendimiento, construcción y posicionamiento como proyecto político, social, ético y epistémico -de saberes y conocimientos-, que afirma la necesidad de cambiar no sólo las relaciones, sino también las estructuras, condiciones y dispositivos de poder que mantienen la desigualdad, inferiorización, racialización y discriminación (Walsh, 2009, p.4).

Nuestro proyecto asume este enfoque, como hilo medular de su praxis . Reconoce y revitaliza saberes ancestrales comunitarios, que cuestionan la problemática global de desigualdad, injusticia social y de depredación de la Naturaleza.

En este contexto, se planteó como un proyecto de innovación, llevado a cabo por un equipo interdisciplinario de docentes de la Universidad Nacional de Educación (UNAE), para el Centro de Educación Infantil (CEI) de innovación anexo a la UNAE, inaugurado el primero de octubre de 2018. Este centro nació de la voluntad de la UNAE de, como su nombre lo indica, crear un espacio de innovación desde la universidad para mejorar la calidad educativa, por medio de nuevas estrategias pedagógicas dentro de la educación pública que pasen por "principios de democratización del conocimiento y de participación colectiva" (Abad, Balfour y Vilanova, 2018, pág. 6).

En cuanto al contexto universitario, esta experiencia ha propiciado una formación integral de los estudiantes; tomando elementos del modelo pedagógico de la UNAE, la acción educativa dentro de la chakra se basa con la propuesta de "teorizar la práctica y experimentar la teoría" (UNAE, 2017, p. 23). De esta manera los contenidos teóricos se articulan con la experiencia, generando un aprendizaje significativo, que se enriquecen desde los enfoques pedagógicos del enactivismo, la pedagogía Waldorf y la educación activa.

El objetivo del proyecto es "crear la chakra escolar en el centro educativo anexo a la UNAE, como ambiente/escenario de aprendizaje transdisciplinar y como núcleo pedagógico 
articulador, en las interacciones UNAE-escuela-familia y comunidad" (AA. VV., 2018, pág. 3). Hemos llevado a cabo esta construcción colectiva desde el nacimiento del CEI, en ella se han planteado debates en torno a metodologías de innovación pedagógicas cooperativas que se nutren de la acción coordinada entre varios actores y de la memoria de los mismos.

\section{Objetivos}

Esta comunicación tiene como objetivo presentar en un primer lugar, los distintos contextos locales en los que hemos tejido esta propuesta; en un segundo momento, los procesos educativos ejecutados por la comunidad de aprendizaje dentro del CEI; y finalmente, las acciones pedagógicas que hemos venido realizando desde la docencia en la UNAE.

\section{Desarrollo de la innovación}

\section{Contextos regionales, locales y los antecedentes de la chakra del CEI de innovación anexo a la UNAE}

En el ámbito de la educación formal, nos interesa el uso de la "chakra" como "didáctica intercultural", y como "ambiente de aprendizaje", en contextos de "educación infantil". Al respecto, hemos encontrado muy poca literatura científica en el periodo comprendido entre 2000 - 2019, en el idioma castellano. Los limitados estudios encontrados en el ámbito educativo sobre la chakra la abordan desde la educación ambiental no formal, y en la educación superior, desde la educación intercultural bilingüe y la agroecología. Sin embargo, no se encuentran estudios, en contextos de educación inicial y superior. La mayor parte de investigaciones encontradas al respecto, se ubican en estudios de tipo antropológico, sobre comunidades indígenas en los Andes, en donde la chakra, ha sido comprendida como un espacio ancestral de relación sagrada con la Pachamama. Considerada también, como un escenario educativo clave, de la revitalización y transmisión de saberes, entre los adultos, niños y niñas.

En Perú, por ejemplo, se ha dado énfasis a la chakra en el ámbito de la educación ambiental intercultural (EAI), en la educación no formal, como vínculo para insertarse en la vida de la comunidad, conviviendo y aprendiendo desde el entorno (GIZ, 2016).

En similar noción y tradición cultural ancestral, en la relación ser humano-naturaleza, se encuentra el concepto de "la milpa", propio de las culturas originarias de Mesoamérica. Méndez (2006), en un análisis en contraposición a la escuela occidental, menciona que la milpa era un lugar educativo para el indígena, pues la educación estaba estrechamente vinculada a la vida y al trabajo. En torno a la milpa, a la cosecha y al procesamiento del maíz, diferentes generaciones de hombres y mujeres transmitían y enriquecían su cultura, además de tener un espacio de encuentro y socialización. La milpa y el contacto con la tierra tenían carácter sagrado, por lo que la vida, la experiencia religiosa, el trabajo y la educación acontecían en un mismo espacio.

Esto nos lleva a inferir una equivalencia conceptual con la chakra de los Andes. En Ecuador, se han registrado dos casos en el ámbito de la educación superior. El primero, se ubica en la Pluriversidad Indígena “Amawtay Wasi” (Institución actualmente vigente), su propuesta 
educativa incorpora a la chakra como eje central de la carrera de agroecología, tomando en cuenta también la memoria ancestral, la historia y la comprensión del manejo local del territorio. Y el segundo, se ubica en la presente experiencia.

En el contexto del sistema educativo ecuatoriano, se plantea la importancia de responder a las necesidades y a los contextos naturales y culturales de los y las estudiantes; es por esta razón que el Modelo del Sistema de Educación Intercultural Bilingüe (MOSEIB) (2013) sostiene la importancia de mantener huertos escolares; sin embargo, estos no se pueden considerar chakras, pues no responden a la cosmovisión andina.

El Ministerio de Educación del Ecuador, con el fin de fortalecer acciones educativas que vinculen a los y las estudiantes con el entorno natural, publicó en 2016 la "Guía Introductoria a la metodología Tierra de niños, niñas y jóvenes para el Buen vivir" (TiNi) (Leguía Orezzoli, 2018). TiNi se le denominó a un espacio desde el metro cuadrado para la creación de huertos escolares. El objetivo de esta acción pedagógica es el de: "promover y fortalecer la cultura y conciencia ambiental en la comunidad educativa mediante la integración y transversalización del enfoque ambiental basado en valores, orientación ética, sentido altruista, innovación y calidad en todo el sistema educativo..." (Ministerio de Educación, 2016, pág. 4). Si bien los elementos ligados a la memoria cultural están poco presentes en este documento, el TINI visibilizó la importancia de la presencia de huertos escolares en espacios educativos.

En el contexto de la UNAE, en el 2016, nació la inquietud por construir un espacio educativo ligado a la tierra. La implementación de la primera chakra (no huerto) parte del proyecto de investigación titulado "Conocimientos ancestrales e innovación social y tecnológica de los/as Agricultores Familiares Campesinos Comunitarios de Cañar, para el diálogo intercultural e intercientífico, en el marco conceptual de la ecología de saberes y el nuevo modelo pedagógico". En este espacio se empezaron a construir aprendizajes desde los enfoques institucionales: "teorizar la práctica y vivenciar la teoría" (UNAE, 2017). Estos aprendizajes se construyeron de manera transdisciplinar durante más de un año (Rosero, Aguilar y Duchi, 2017). Posteriormente se fortaleció la propuesta de otra chakra en el grupo académico (no oficial) "Pachasofía de la Chakra" y finalmente, la retomamos desde el grupo de innovación interdisciplinario "La Chakra escolar, como núcleo pedagógico articulador y ambiente de aprendizaje transdisciplinar, aplicado en la Unidad Educativa anexa a la UNAE", el mismo que es parte del grupo de investigación institucional "Tierra: Educación, Ambiente e Interculturalidad".

En relación al CEI de innovación de la UNAE, su modelo pedagógico se alinea a la propuesta de la chakra, pues define a este centro como un espacio educativo que busca potenciar "las competencias humanas, el trabajo cooperativo y comunitario de los estudiantes y que responda a sus necesidades directas y en correspondencia al contexto donde pertenecen, con una visión ancestral de sus raíces y una proyección internacional de su interculturalidad" (Abad, Balfour y Vilanova, 2017).

Estos contextos tanto nacionales como institucionales constituyeron un escenario propicio para la implementación del proyecto de innovación de la chakra que ha tenido como propósito construir conocimientos desde el aprendizaje colaborativo y desde la comunidad de aprendizaje. 


\section{La chakra, semillero de innovación y de comunidades de aprendizaje}

Este proyecto se concibe a sí mismo como un ambiente de aprendizajes transdisciplinarios construido sobre la base de una comunidad de aprendizaje. Dentro de este colectivo se articulan las acciones pedagógicas de: las y los docentes-investigadores de la UNAE, las y los profesores del CEI (dos de ellos son parte del Ministerio de Educación y ocho son también docentes de la UNAE), las familias y los niños y niñas de esta institución y los y las estudiantes de la UNAE de diferentes carreras y asignaturas.

La experiencia en la chakra está llena de constantes aprendizajes acerca de cómo se construye el conocimiento desde los saberes colectivos, surgidos en el devenir de conversaciones con los diversos actores que están en relación con la misma. Cada uno de las y los actores tienen distintos roles; sin embargo, todos convergen a un mismo fin: crear y mantener el espacio de la chakra a través del trabajo cooperativo-horizontal basado en los conocimientos ancestrales propios de la zona. Nuestros encuentros han buscado tejer y revitalizar saberes ancestrales dentro de la comunidad UNAE-escuelita, develando que estos conocimientos están arraigados a la cultura y que están presentes en cualquiera de nosotras y nosotros.

La implementación de este proyecto inició previo a la inauguración del centro educativo. Durante la primera reunión con las familias de los infantes del centro presentamos al equipo de investigadores y el plan de trabajo que se tenía, acordamos la primera minga para la preparación del terreno, la limpia. Asimismo, hemos desarrollado diversas actividades de aprendizaje y celebración en torno a la chakra como se describe a continuación.

\subsection{La limpia del espacio}

A tres semanas de inaugurado el CEI de innovación, organizamos la primera minga colectiva para la limpia. Contamos con la presencia de familiares de los niños y niñas del centro, con algunos de los niños y niñas, los docentes investigadores de este proyecto, estudiantes de la UNAE y algunos docentes del CEI.

La primera actividad consistió en hacer un ritual para pedir permiso a la tierra - en kichwa (lengua ancestral propia de la zona) y en español - para la limpia y la siembra. Posteriormente compartimos con la tierra la chicha (una bebida tradicional ecuatoriana hecha a base de maíz). Este ritual responde a la cosmovisión del mundo andino, en la cual la tierra no es un objeto inerte, sino que es la fuente primordial de la vida, es la madre tierra, la pacha mama.

Como proceso educativo, cada uno de los padres, madres y docentes narraron frente a las y los niños sus experiencias y recuerdos ligados a la tierra y su siembra. Luego pasamos a la limpia del terreno con los picos. Los niños y niñas también apoyaron con picos pequeños. Se consiguió limpiar toda la zona cultivable, entre relatos de siembras, bromas y chicha.

Al cierre de la jornada de trabajo, realizamos una pampamesa, un espacio colectivo ancestral de comida en el cual cada participante de la minka (encuentro de trabajo cooperativo) trae un alimento y lo comparte con el resto. Se instalan manteles en el piso y sobre los manteles se pone toda la comida. Cada uno coge libremente lo que quiera comer. 


\subsection{El proceso cotidiano de aprendizaje con los infantes}

Actualmente, la asignatura del CEI que ha estado directamente vinculada a la chakra es la de kichwa, aunque, en esta segunda fase del proyecto, nuestro objetivo es capacitar a las y los docentes del centro para que puedan identificar las potencialidades de la chakra y utilizarla más dentro de sus planificaciones de clases cotidianas.

El aprendizaje de esta lengua ancestral se da en contextos culturales ligados a la tierra y al cultivo. Los niños y niñas aprenden a contar en kichwa, sobre las plantas, los colores y otros contenidos, mientras preparan las semillas, las identifican, separan y siembran. Asimismo, en la cosecha se han trabajado contenidos sobre colores, tamaños y sabores (Fig. 1).

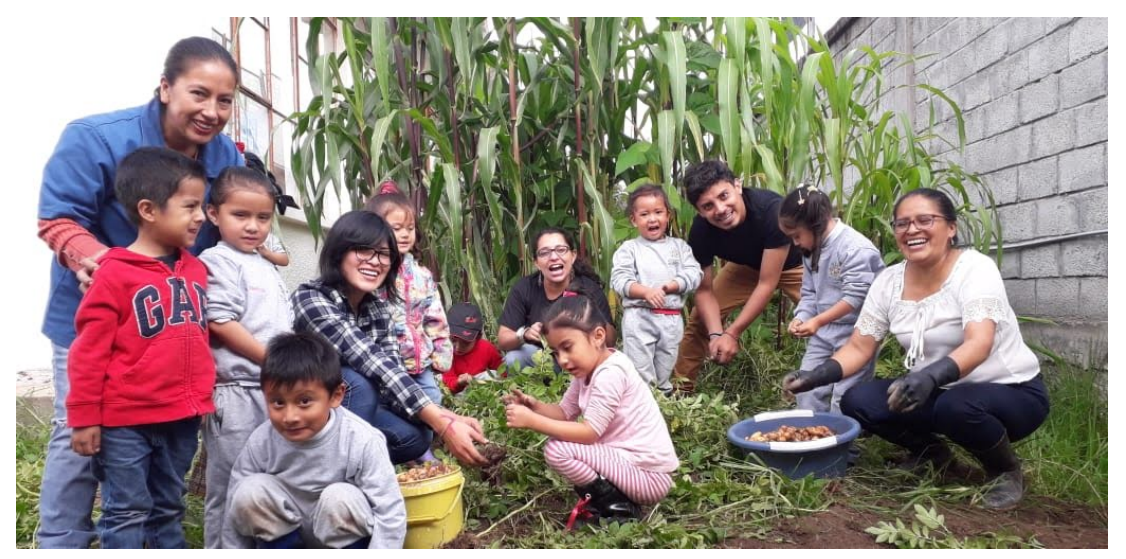

Fig. 1 Minka de cosecha de papas en la Chakra Fuente: autores del artículo

El momento de la siembra fue clave en el CEI, la clase con alumnos de inicial 1 (tres años) y con inicial 2 (cuatro años), partió de hablar sobre las plantas, lo que significan para nosotros como fuente de alimento y la importancia de cuidarles y respetarles. Posteriormente, pasamos a la siembra, en la que hablamos de las características de cada semilla y contamos en kichwa el número de cada una (dos de maíz y tres de fréjol). Este tipo de siembra responde a las técnicas ancestrales de asociatividad. A parte de esto, trabajamos otros contenidos como distancias entre cada uno de los hoyos sembrados, psicomotricidad fina y gruesa. Se cerró la actividad con una canción sobre el cuidado de las plantas. Durante estos cuatro meses, hemos podido constatar que las niñas y los niños han desarrollado respeto por las plantas y las asumen como sus "hijas", lo que consideramos como uno de los principales logros de este proyecto.

Para el mantenimiento de este espacio se emplea un sistema de corresponsabilidad con la familia de los niños, los investigadores y unos actores claves: los estudiantes de la UNAE, tal como se lo explicará en el siguiente apartado.

\subsection{La chakra, el calendario agrofestivo y las fiestas}

La chakra tiene su propio tiempo, no se puede sembrar en cualquier momento. Cada proceso tiene su etapa, con relación a los fenómenos de la naturaleza y al clima. Si se siembra en otras 
épocas, el exceso o la falta de lluvias arruinaría la cosecha. Estos conocimientos son propios de la sabiduría ancestral. Por esta razón, para la implementación de este proyecto hemos utilizado el calendario agrofestivo del pueblo cañari, que establece meses de siembra, cosechas, deshierbe, limpias.

Otra de las temporalidades a ser tomadas en cuenta son las celebraciones kichwas de los raymis (fiesta en kichwa) que corresponden a los días de solsticios y de equinoccios. Estas fechas han sido incorporadas en el calendario académico del centro de educación. De esta manera, mientras el 21 de diciembre se festejaba navidad; también se celebró el Kapak Raymi, solsticio que marca nuevos ciclos.

Desde esta perspectiva logramos trabajar el diálogo de saberes, la decolonización epistémica que reconoce tanto los conocimientos de occidente, como los ancestrales, y los enfoques de interculturalidad con toda la comunidad educativa: los niños, niñas y sus familias, los docentes del CEI, los investigadores de la UNAE y los estudiantes de la universidad.

En definitiva, el proyecto de la Chakra se ha implementado por medio de la consolidación de una comunidad de aprendizaje. A continuación, abordaremos el proceso visto desde la perspectiva de la universidad.

\section{Desarrollo de la Experiencia (Escolar y Universidad UNAE)}

Dentro de este proyecto se ha planteado que la chakra se establezca como un núcleo pedagógico articulador en las interacciones UNAE-Escuela, por ello, estudiantes de las carreras de Educación Intercultural Bilingüe (EIB), Educación inicial (EI) y Educación en Ciencias Experimentales (ECE) de la UNAE se han involucrado activamente en la experiencia. El enfoque central que se le ha dado a esta propuesta de innovación educativa ha sido la creación de un ambiente de aprendizaje fuera del aula en donde se propicia un proceso de enseñanza-aprendizaje vivencial. Se han articulado elementos del enactivismo, el cual resalta la importancia de conocer en y para la acción a través de la interacción corporal, experiencial y cognitiva (Varela, Thompson \& Rosch, 1992).

Desde la pedagogía Waldorf se inculca a los alumnos que el aprendizaje es sistémico y que es indispensable que la familia, la comunidad y las y los docentes confluyan en acciones educativas conjuntas. Desde el trabajo en la chakra se desarrolla la voluntad y no solamente de contenidos académicos lo cual también se alinea con esta escuela pedagógica (Rodríguez Palacios, 2013, p. 24).

Hemos desarrollado actividades de reflexión e interacción dentro de la chakra como un ambiente de aprendizaje, generándose un intercambio de saberes con las y los estudiantes sobre su conocimiento alrededor de las plantas cultivadas comúnmente en sus lugares de origen, los calendarios agrofestivos y las prácticas de siembra. Asimismo, hemos realizado minkas en la chakra para la limpieza del terreno, el deshierbe y aporque y la construcción de una compostera.

Desde la asignatura de Cátedra integradora: Sistemas educativos, la cual realiza una aproximación a la política pública en Educación y del Buen Vivir, hemos trabajado con las y los estudiantes de ECE sobre el potencial de la chakra para articular las políticas señaladas 
en la Constitución de la República del Ecuador, la Ley Orgánica de Educación Intercultural y el MOSEIB con la práctica educativa. Además, han surgido diferentes iniciativas de estrategias pedagógicas para el desarrollo de los contenidos de asignaturas como Ciencias naturales, Química, Física, Matemáticas y Estudios Sociales en torno a la chakra. Asimismo, hemos discutido sobre la importancia de la misma como un espacio de contacto con el medio natural en el que las y los actores desarrollan valores como el respeto y la sensibilidad por el medio ambiente.

Asimismo, desde el aprendizaje significativo y la pedagogía activa se está trabajando actualmente en el desarrollo de proyectos para aprovechar el espacio de la Chakra como un entorno de enseñanza-aprendizaje y trabajar los contenidos del Currículum de la asignatura de Ciencias Naturales como dinámica de los ecosistemas, Sistemas de vida y transferencia entre materia y energía. Además se han abordado las temáticas de soberanía alimentaria y se ha trabajado el tema de Educación ambiental de forma transversal.

\section{Conclusiones}

La chakra, entendida como estrategia pedagógica, al igual que experiencias previas de implementación de huertos escolares en otros contextos (Eugenio Gozalbo \& Aragón Núñez, 2016; Moncada, 2017), contribuye al desarrollo de los contenidos de diferentes asignaturas desde un enfoque de interacción con el entorno natural.

Esta iniciativa pedagógica rebasa la propuesta del TINI (sobre todo en su forma de aplicarse en la práctica), ya que trabajamos la tierra desde los conocimientos ancestrales locales como son el caledario agrofestivo, la agricultura asociativa y orgánica. La visión del proyecto garantiza la participación activa de los padres y las madres de familia, con lo que se genera una trasmisión intergeneracional del conocimiento.

La chakra del CEI de innovación anexo a la UNAE constituye un ambiente de aprendizaje que deben partir de un enfoque transdisciplinar desde el cual los infantes pueden desarrollar las competencias de los distintos ámbitos presentes en el curriculum de educación inicial ecuatoriano. El éxito de esta experiencia de innovación radica no solo en la implementación metodológica, en la cual ya se construye un proceso educativo; sino en que el resto de la planta docente pueda comprender las cosmovisiones que la encierran para planificar actividades en este espacio.

Además, este proyecto parte de la construcción de una comunidad de aprendizaje, en la cual se teje de manera horizontal una red de conocimientos entre la familia, los docentes y las y los miembros del proyecto. Las decisiones sobre la forma de construir este espacio se toman de manera colaborativa, en la que todas las voces son importantes. Las y los alumnos tanto de la UNAE, como del CEI de innovación son los beneficiaros directos de este proceso educativo, ya que, tal como se indica en el modelo pedagógico de la universidad, para conseguir un aprendizaje significativo se debe "teorizar la práctica y vivenciar la teoría" (UNAE, 2017).

Finalmente, la participación de todas y todos en el proyecto ha forjado diversas dinámicas de relación entre compañeros y compañeras y ha propiciado un clima de colaboración, diálogo 
y respeto a los saberes de las y los otros. El contacto con la tierra ha permitido un aprendizaje vivencial. Las y los actores han desarrollado un vínculo de compromiso con la Chakra y su cuidado, a través del respeto y el amor. Siendo así, entonces, un espacio que llama a compartir $\mathrm{y}$ aportar entre todas y todos $\mathrm{y}$, donde se mueven emocionalidades y se construyen lazos tanto con el entorno como entre los diversos actores que participan de su cuidado. En definitiva, la Chakra genera intercambios alrededor de los saberes ancestrales y la revalorización de la cultura, la memoria y la identidad local.

\section{Referencias}

AA.VV. (2018). "La Chakra escolar, como núcleo pedagógico articulador y ambiente de aprendizaje transdisciplinar, aplicado en la Unidad Educativa anexa a la UNAE”. Azogues: UNAE.

ABAD J., BALFOUR B., Vilanova M. (2017). "Propuesta de innovación pedagógica: unidad de innovación educativa anexa a la UNAE”. Azogues: UNAE.

AGUILAR, J., DUCHI, A., y ROSERO, F. (2017). “La huerta UNAE, un espacio pedagógico innovador". Mamakuna, (2017, 63). Azogues: UNAE.

AREVALO, M. (2017). "La chakra y el mundo andino". Cidap, 6. Recuperado de: http://documentacion.cidap.gob.ec:8080/bitstream/cidap/1642/1/La\%20chakra\% 20y\%20el\%20mundo\%20andino.pdf

EUGENIO, M., \& ARAGÓN, L. (2016). "Experiencias en torno al huerto ecológico como recurso didáctico y contexto de aprendizaje en la formación inicial de maestros de Infantil”. Revista Eureka Sobre Enseñanza y Divulgación de las Ciencias, (2016, 3, 13, 667-679).

GIZ, Cooperación Alemana, (2016). "Guía para la contextualización de la Educación Ambiental Intercultural”. Tercera edición. Ediciones Nova Print Sac: Lima.

LEGUÍA OREZZOLI, J (2018). Guía introductoria a la metodología TiNi (2nd ed.). Quito: Ministerio de Educación del Ecuador.

MENDEZ, M. (2006). “Educación, control social y emancipación”. Teoría y praxis. (No. 9. p. 106-121).

Ministerio de educación del Ecuador (2013). Modelo del Sistema de Educación Intercultural Bilingüe. Quito: Ministerio de educación.

Ministerio de educación del Ecuador (2016). Guía Introductoria a la metodología Tierra de niños, niñas y jóvenes para el Buen vivir. Quito: Ministerio de educación.

MONCADA, S. (2017). La huerta escolar agroecológica como ambiente de aprendizaje colaborativo en el Colegio Monseñor Ramón Arcila del Carmen de Viboral. Trabajo final de titulación. Antioquia: Universidad de Antioquia.

RODRÍGUEZ PALACIOS, S. (2013). Investigación sobre los orígenes y la aplicación de la pedagogía Waldorf. Palencia: Escuela Universitarida de Educación. Campus Palencia.

ROSERO, AGUILAR y DUCHI (2017). "La huerta UNAE, un espacio pedagógico innovador”. Mamakuna (2017, $\left.\mathrm{n}^{\circ} 6\right)$. Azogues: UNAE.

Universidad Nacional de Educación. (2017). Modelo Pedagógico de la Universidad Nacional de Educación del Ecuador. Azogues: UNAE.

(c) EY-NC-ND 2019, Universitat Politècnica de València 
VARELA, F., THOMPSON, E. y ROSCH (1992). De cuerpo presente. Las ciencias cognitivas y la experiencia humana. Barcelona: Gedisa.

Walsh, C. (2009). "Interculturalidad crítica y educación intercultural”. Ponencia presentada en el Seminario "Interculturalidad y Educación Intercultural". La Paz: Instituto Internacional de Integración del Convenio Andrés Bello. 